\title{
Selection of Antibody Responses Associated With Plasmodium falciparum Infections in the Context of Malaria Elimination
}

\author{
Lotus L. van den Hoogen ${ }^{1,2 \star}$, Gillian Stresman ${ }^{1}$, Jacquelin Présumé ${ }^{3}$, Ithamare Romilus ${ }^{3}$, \\ Gina Mondélus ${ }^{3}$, Tamara Elismé ${ }^{3}$, Alexandre Existe ${ }^{3}$, Karen E. S. Hamre ${ }^{4,5}$, \\ Ruth A. Ashton ${ }^{2}$, Thomas Druetz ${ }^{2,6}$, Vena Joseph ${ }^{2}$, James G. Beeson ${ }^{7,8,9}$, \\ Susheel K. Singh ${ }^{10,11}$, Jacques Boncy ${ }^{3}$, Thomas P. Eisele ${ }^{2}$, Michelle A. Chang ${ }^{4}$, \\ Jean F. Lemoine ${ }^{12}$, Kevin K. A. Tetteh ${ }^{1}$, Eric Rogier ${ }^{4}$ and Chris Drakeley ${ }^{1}$

\begin{abstract}
${ }^{1}$ Department of Infection Biology, London School of Hygiene \& Tropical Medicine, London, United Kingdom, ${ }^{2}$ Center for Applied Malaria Research and Evaluation, Tulane University School of Public Health \& Tropical Medicine, New Orleans, LA, and Malaria, Centers for Disease Control and Prevention, Atlanta, GA, United States, ${ }^{5}$ CDC Foundation, Atlanta, GA, Canada, ${ }^{7}$ Burnet Institute, Melbourne, VIC, Australia, ${ }^{8}$ Department of Medicine, The University of Melbourne, Melbourne, VIC, Australia, ${ }^{9}$ Central Clinical School and Department of Microbiology, Monash University, Clayton, VIC, Australia, ${ }^{10}$ Department of Congenital Disorders, Statens Serum Institut, Copenhagen, Denmark, ${ }^{11}$ Department of Immunology and Microbiology, Centre for Medical Parasitology, University of Copenhagen, Copenhagen, Denmark, ${ }^{12}$ Ministère de la Santé Publique et de la Population, Port-au-Prince, Haiti
\end{abstract} \\ United States, ${ }^{3}$ Laboratoire National de Santé Publique, Port-au-Prince, Haiti, ${ }^{4}$ Malaria Branch, Division of Parasitic Diseases \\ United States, ${ }^{6}$ Department of Social and Preventive Medicine, University of Montreal School of Public Health, Montreal, QC,
}

\section{OPEN ACCESS}

Edited by:

Wanderley De Souza

Federal University of Rio de

Janeiro, Brazi

Reviewed by:

Luiz Gustavo Gardinassi,

Universidade Federal de Goiás

(IPTSP - UFG), Brazil

Sunil Joshi,

University of Miami - Miller School of

Medicine, United States

*Correspondence:

Lotus L. van den Hoogen

Ivandenhoogen@tulane.edu

Specialty section:

This article was submitted to Microbial Immunology,

a section of the journal

Frontiers in Immunology

Received: 28 February 2020

Accepted: 21 April 2020

Published: 15 May 2020

Citation:

van den Hoogen LL, Stresman G,

Présumé J, Romilus I, Mondélus G,

Elismé T, Existe A, Hamre KES,

Ashton RA, Druetz T, Joseph V,

Beeson JG, Singh SK, Boncy J,

Eisele TP, Chang MA, Lemoine JF,

Tetteh KKA, Rogier E and Drakeley C

(2020) Selection of Antibody

Responses Associated With

Plasmodium falciparum Infections in

the Context of Malaria Elimination.

Front. Immunol. 11:928.

doi: 10.3389/fimmu.2020.00928
In our aim to eliminate malaria, more sensitive tools to detect residual transmission are quickly becoming essential. Antimalarial antibody responses persist in the blood after a malaria infection and provide a wider window to detect exposure to infection compared to parasite detection metrics. Here, we aimed to select antibody responses associated with recent and cumulative exposure to malaria using cross-sectional survey data from Haiti, an elimination setting. Using a multiplex bead assay, we generated data for antibody responses (immunoglobulin G) to 23 Plasmodium falciparum targets in 29,481 participants across three surveys. This included one community-based survey in which participants were enrolled during household visits and two sentinel group surveys in which participants were enrolled at schools and health facilities. First, we correlated continuous antibody responses with age (Spearman) to determine which showed strong age-related associations indicating accumulation over time with limited loss. AMA-1 and MSP-1 19 antibody levels showed the strongest correlation with age $(0.47$ and $0.43, p<0.001)$ in the community-based survey, which was most representative of the underlying age structure of the population, thus seropositivity to either of these antibodies was considered representative of cumulative exposure to malaria. Next, in the absence of a gold standard for recent exposure, we included antibody responses to the remaining targets to predict highly sensitive rapid diagnostic test (hsRDT) status using receiver operating characteristic curves. For this, only data from the survey with the highest hsRDT prevalence was used $(7.2 \% ; 348 / 4,849)$. The performance of the top two antigens in the training dataset (two-thirds of the dataset; 
$n=3,204$ )-Etramp 5 ag 1 and GLURP-RO (area-under-the-curve, AUC, 0.892 and 0.825 , respectively) - was confirmed in the test dataset (remaining one-third of the dataset; $n=1,652$, AUC 0.903 and 0.848 , respectively). As no further improvement was seen by combining seropositivity to GLURP-RO and Etramp 5 ag 1 ( $p=0.266$ ), seropositivity to Etramp 5 ag 1 alone was selected as representative of current or recent exposure to malaria. The validation of antibody responses associated with these exposure histories simplifies analyses and interpretation of antibody data and facilitates the application of results to evaluate programs.

\section{Keywords: malaria, immunoglobulin G (IgG), multiplex bead assay, sero-surveillance, elimination, ETRAMP}

\section{INTRODUCTION}

Globally, the number of countries in malaria pre-elimination and elimination phases is increasing (1). For malaria control and elimination purposes, the allocation of national resources is commonly guided by regional case counts. However, trends in passively detected cases are dependent on health system coverage and treatment-seeking behavior, and thus may not

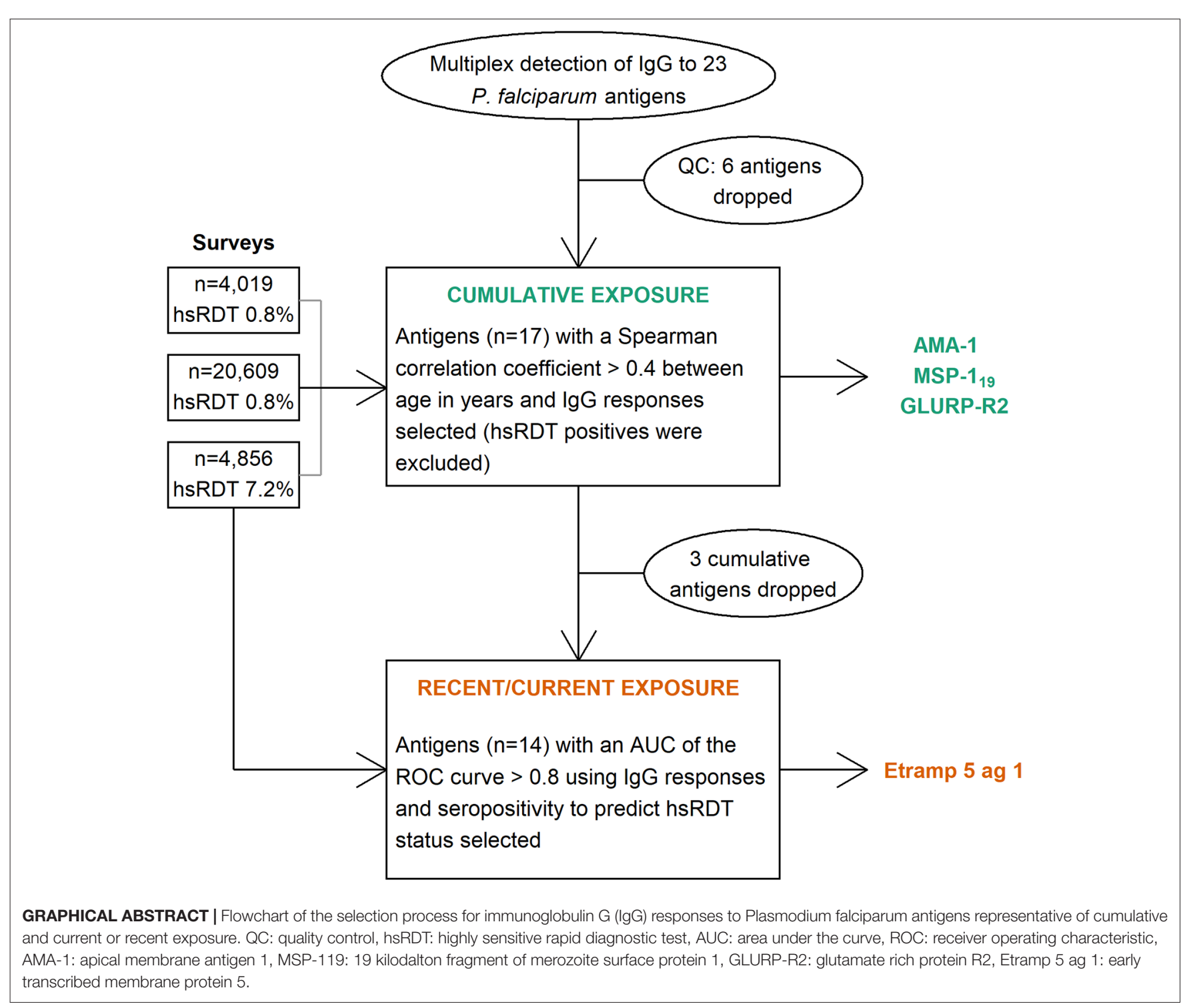


represent the true malaria burden in all settings. Serological endpoints, typically the presence of antibody responses in resident populations, represent a population's exposure history and can identify areas with residual malaria transmission more accurately than the prevalence of infection at low transmission (2).

Until recently, sero-surveillance has primarily focused on antibodies representing cumulative exposure, such as Plasmodium falciparum apical membrane antigen 1 (AMA1) and the $19 \mathrm{kDa}$ fragment of merozoite surface protein 1 $\left(\mathrm{MSP}_{1} \mathrm{1}_{19}\right)$ (2). Age-specific increases in seroprevalence to these antigens, estimated as seroconversion rates (SCR), have been shown to be strongly correlated with entomological inoculation rates (EIR), the gold standard metric for transmission intensity, and with parasite prevalence (2). Antibodies to these antigens persist in the blood with repeated exposure. For MSP-1 19 , model estimates suggested the time to sero-reversion is 23 (3) to 50 years or more (4), while limited data from observational studies suggest half-lives of long-lived antibody secreting cells to be 2 (5) to 16 (6) years. Although MSP-1 19 and AMA-1 antibody half-lives might be faster in children $(7,8)$, this may be due to insufficient repeated exposure in children at low transmission. Antibodies with shorter half-lives [i.e., those indicating incidence in the past year (9)] may be able to detect if and where changes in malaria transmission intensity take place more rapidly and accurately as compared to antibodies with long half-lives. Several potential candidates have recently been optimized for use in multiplex bead assays (MBA) (10).

As part of Haiti's aim to eliminate malaria (11), largescale cross-sectional surveys were performed to assess if and where residual transmission, potentially undetected via routine surveillance, is occurring. Here, we assessed antibody responses to $23 P$. falciparum recombinant proteins and peptides in 29,481 participants residing in two areas with different levels of transmission intensity. Our aim was to select antibodies associated with cumulative and current or recent exposure to malaria for the Haitian context in order to simplify analyses and interpretation of collected survey data that can be used to inform program decisions.

\section{METHODS}

\section{Study Population}

The island of Hispaniola, consisting of Haiti and the Dominican Republic, is the last remaining region in the Caribbean with malaria transmission. In $2016,97 \%$ of the reported malaria cases on the island occurred in Haiti (12). In Haiti, transmission is highly focal as the Grand'Anse department, in the southwestern part of the country, accounted for $47 \%$ of the national malaria cases reported in 2017 (13). Three large-scale cross-sectional surveys were conducted in Haiti in 2017 (14): (1) in Artibonite, central Haiti, a community-based household survey (HHArtibonite) with 21,891 participants; (2) an easy-access-group (EAG) survey in the same area (EAG-Artibonite) including 6,006 participants; and (3) an EAG survey was performed with the same protocol in Grand'Anse, southwestern Haiti, (EAG-Grand'Anse) with 5,034 participants (Table 1). For the
EAG surveys, participants were enrolled at schools and health facilities. Although in EAG-Artibonite participants were also sampled at church venues, these were excluded from further analyses due to practical and logistical restraints of this sentinel group (16). At health facilities, participants were enrolled irrespective of the reason for their visit, and both treatmentseeking patients as well as accompanying people were asked to participate. In the HH-Artibonite survey, households were randomly selected in a defined geographic area and all household members were asked to participate; participants were tested at their homes (Hamre et al., in preparation). In both survey types, people were asked to participate irrespective of the presence of fever or symptoms of malaria. Participants from all three cross-sectional surveys provided finger-prick blood for malaria diagnosis by a conventional rapid diagnostic test (cRDT; SD Bioline Malaria Antigen P.f., 05FK50, Standard Diagnostics) and a highly-sensitive RDT (hsRDT; also known as ultrasensitive RDT, Alere Malaria Ag P.f., 05FK141, Standard Diagnostics). In addition, finger-prick blood was spotted on Whatman 903 cards (GE Healthcare), dried overnight at ambient temperature and packed the next day with silica gel. Dried blood spots (DBS) were stored at $+4^{\circ} \mathrm{C}$ and transported to the national laboratory (Laboratoire National de Santé Publique; LNSP) in Port-au-Prince weekly. Demographic and household characteristics were collected through a tablet-based questionnaire. Participants testing positive by cRDT were treated according to national guidelines.

\section{Antigen Panel and Covalent Coupling of Antigens to Beads}

Twenty-three P. falciparum antigens and peptides from asexual life-cycle stages were included in the panel (Table 2). Antigens were covalently coupled to unique bead regions as described by Rogier et al. (34). Details on antigen to bead coupling conditions are described elsewhere for most of the antigens in the panel (15), while for the remaining six antigens these are provided in Supplementary Table 1.

\section{Antimalarial Antibody Detection}

Immunoglobulin G (IgG) data were collected for all participants as previously described (34). Antibody levels were measured using a MBA with a OneStep protocol enabling a rapid turnaround by incubating sample and secondary antibody simultaneously overnight (34). Median fluorescence intensity (MFI) was recorded using the MAGPIX with Bio-Plex Manager ${ }^{\mathrm{TM}}$ MP software (BioRad) and corrected for background reactivity to give a final value of MFI minus background (hereafter: MFI). Alongside participant samples, a 6-point serial dilution of a hyperimmune positive control pool (i.e., standard curve) was added to each plate to assess plate-to-plate variation in data collection.

\section{Quality Control of Antimalarial Antibody Data Collection}

Plates with results that fell outside of the mean $+/-2$ standard deviations (SD) of the third point of the serial dilution for two out 
TABLE 1 | General characteristics of the study population for three malaria cross-sectional surveys performed in Haiti.

\begin{tabular}{|c|c|c|c|}
\hline Characteristic & HH-Artibonite & EAG-Artibonite & EAG-Grand'Anse \\
\hline Department & Artibonite, central Haiti & Artibonite, central Haiti & Grand'Anse, southwestern Haiti \\
\hline Communes & Verrettes \& La Chappelle & Verrettes \& La Chappelle & $\begin{array}{l}\text { Anse-d'Hainault, Chambellan, Dame-Marie, } \\
\text { Les Irois \& Moron }\end{array}$ \\
\hline Timing & Jul-Oct $2017^{\star}$ & May-Jun 2017 & Nov-Dec 2017 \\
\hline Survey type & $\begin{array}{l}\text { Community-based } \\
\text { household survey }\end{array}$ & $\begin{array}{l}\text { Easy-access-group survey: participants } \\
\text { recruited in churches, schools \& health facilities }\end{array}$ & $\begin{array}{l}\text { Easy-access-group survey: participants } \\
\text { recruited in schools \& health facilities }\end{array}$ \\
\hline \multicolumn{4}{|l|}{$\mathrm{N}$} \\
\hline - Available lgG data** & 21,235 & 5,898 & 4,967 \\
\hline - Merged to field data & 21,214 & $4,154^{\star \star \star}$ & 4,959 \\
\hline - Aged $\geq 1$ year & 20,609 & 4,019 & 4,856 \\
\hline $\begin{array}{l}\text { Median age, } \\
\text { interquartile range }\end{array}$ & $20,8-41$ & $12,8-24$ & $13,8-22$ \\
\hline $\begin{array}{l}\text { Highly sensitive RDT } \\
\text { prevalence, } n / N\end{array}$ & $0.76 \%, 157 / 20,556$ & $0.77 \%, 31 / 4,019$ & $7.18 \%, 348 / 4,849$ \\
\hline
\end{tabular}

RDT: rapid diagnostic test. IgG: immunoglobulin G.

${ }^{*}$ Two-week pause due to hurricanes.

${ }^{* \star}$ For more information on the number samples for which IgG data was successfully collected see van den Hoogen et al. (15).

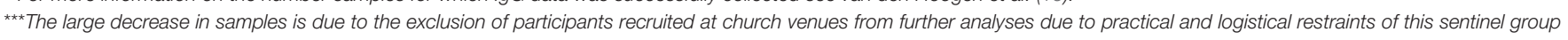
(16).

of three highly immunogenic antigens (GLURP-R2, AMA-1 and MSP-1 ${ }_{19}$ ) were repeated (15). Of the twenty-three P. falciparum antigens and peptides included in the panel, six had to be excluded as they showed loss in reactivity over time (previously described in van den Hoogen et al. (15) and for the remaining six antigenic targets shown in Supplementary Figure 1): MSP2_Dd2, MSP2_CH150/9, GEXP18, EBA-140 RIII-V, Rh2_2030, and Rh4.2. The remaining seventeen antigenic targets were analyzed for their association with cumulative and current or recent exposure to falciparum malaria.

\section{Statistical Analyses Data Standardization}

All statistical analyses were performed in $\mathrm{R}$ version 3.4.1 (35). After correction for background reactivity, the lowest MFI value recorded was-61, thus 65 was added to all values (i.e., for all antigens across all participants) and resulting MFI values were $\log _{10}$-transformed. Participants with high IgG responses to glutathione S-transferase (GST) were removed from further analyses (MFI $>1,000)$ (15). In addition, results from participants below the age of 1 were excluded from the dataset to remove any influence of maternally derived antibodies. Finite mixture models were used to identify two components in the $\log _{10}$ (MFI) participant data and the lower distribution was assumed to consist of seronegative individuals. Thresholds for seropositivity were defined as the mean of the lower distribution plus 5 SD (for details see Supplementary Methods in Supplementary Data Sheet 2).

\section{Antibody Responses Associated With Cumulative Exposure}

Antibody responses ( $\log _{10}$ MFIs) from participants were correlated with age; those that were most strongly correlated with age were considered to represent cumulative exposure. Analysis was conducted using the continuous antibody data instead of seropositivity to maximize the strength of any correlation. The $\mathrm{HH}$-Artibonite survey was considered most informative in assessing correlations between antibodies and age, as it represents the full age-structure of the population sampled due to its community-based sampling strategy compared to sentinel group sampling in the EAG-surveys (children attending school and healthcare-seeking populations sampled at local facilities). Participants who were hsRDT positive were excluded to remove any antibody responses due to a current or recent infection. Spearman correlation coefficients ( $r$ ) between all antibody responses and age (in years) were plotted using the $\mathrm{R}$ package corrplot (36). Only pairwise complete observations were used. The Bonferroni correction for multiple-comparisons was used to adjust the p-value for statistical significance. The chi-squared test for trend in proportions was used to test age-specific trends in seroprevalence [stats package in $\mathrm{R}(35)]$.

\section{Antibody Responses Associated With Current or Recent Exposure}

In absence of a gold standard for recent exposure (i.e., confirmed recent infection), hsRDT was used to determine antibody responses associated with current or recent exposure. The hsRDT detects histidine-rich protein 2 (HRP2), a P. falciparum antigen. HRP2 has been shown to persist in the blood up to weeks after parasite clearance following effective antimalarial treatment (37). The hsRDT has a lower limit of detection for HRP2 antigenemia (40-125 pg/mL HRP2) compared to the cRDT (600-1000 pg/mL HRP2) (38) and is thus potentially more sensitive to (chronic) low-density infections producing only low levels of HRP2. A positive hsRDT result may therefore indicate a current or recent 
TABLE 2 | Characteristics of multiplex bead assay Plasmodium falciparum antigen panel.

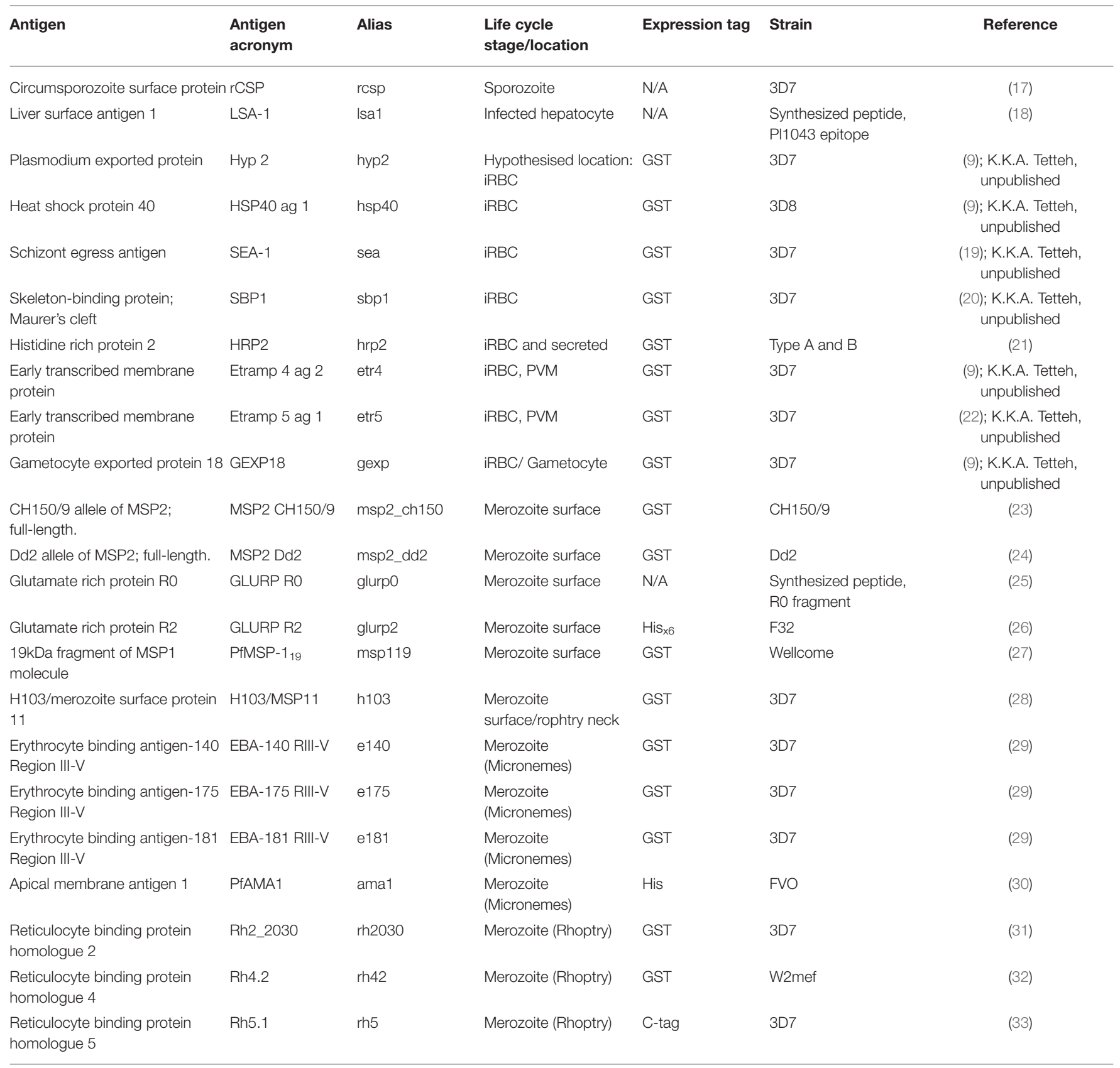

MSP, merozoite surface protein; kDa, kilodalton; GST, Glutathione S-transferase; iRBC, infected red blood cell; PVM, parasitophorous vacuole membrane.

infection. Antibody responses to antigenic targets that were not selected as cumulative exposure markers, were used to predict hsRDT positivity using receiver operating characteristic (ROC) curves. To assess utility to predict hsRDT infection status, only participant data from EAG-Grand'Anse was used, which was the survey with the highest rate of hsRDT positives. The EAGGrand'Anse data was split into a training dataset (two-third, $n$ $=3,201$ ) and a test dataset (one-third, $n=1,648$ ) using random selection. The numbers of hsRDT positive samples in the training dataset was $227(7.1 \%)$ and in the test dataset was $120(7.3 \%)$.
The area-under-the-curve (AUC) of ROCs [ $p R O C$ package; (39)] was measured using the training dataset in all ages as well as separately for those aged 1 to 15 years and those aged older than 15 years to assess any differences by age. The performance of the most informative antibody responses in predicting hsRDT status (i.e., AUC >0.8) was confirmed by repeating ROCs for the test, HH-Artibonite, and EAG-Artibonite datasets. Finally, ROCs using seropositivity endpoints were also assessed in the test dataset. ROCs were compared using DeLong's test for two correlated ROC curves [roc.test within the pROC package; (39)]. 


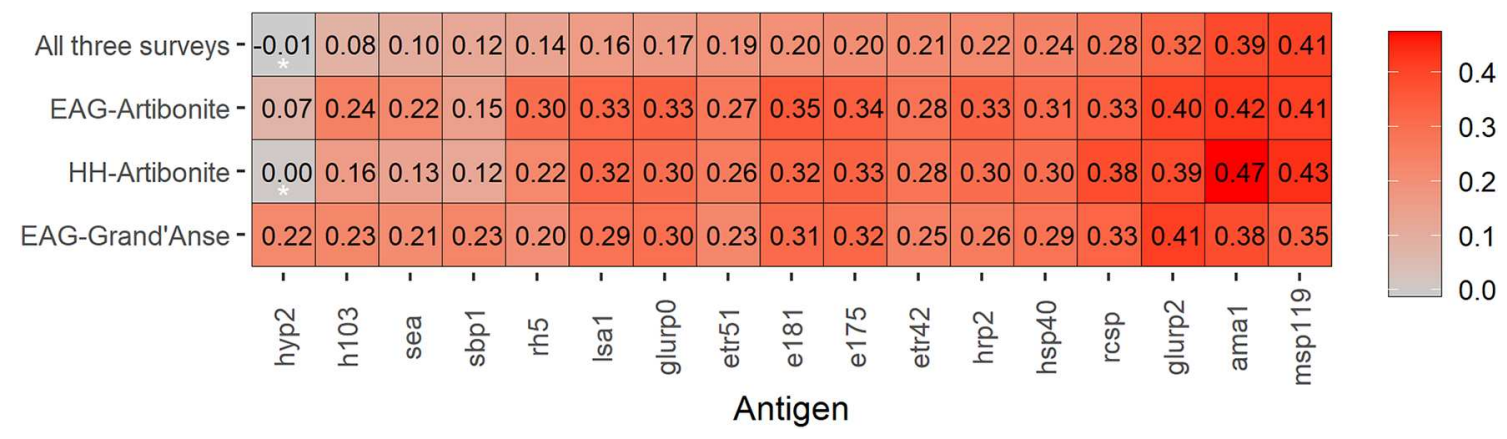

FIGURE 1 | Heat map of Spearman correlation coefficients for age and immunoglobulin G (lgG) responses to seventeen Plasmodium falciparum antigens across three malaria transmission surveys in Haiti. IgG responses were defined as log10-transformed median fluorescence intensities corrected for background reactivity, while age is in years. Data is shown for 28,888 participants; highly sensitive rapid diagnostic test (hsRDT) positives were excluded ( $n=536$ ) to remove any lgG responses due to a current or recent infection. Coloring represents the strength of Spearman correlation coefficients from weak in grey to strong in red. Antigens ( $\mathrm{x}$-axis) are ordered from the lowest to highest Spearman correlation coefficient using data from all three surveys. Spearman correlation coefficients that were not statistically significant (i.e., $p>0.003$ assuming Bonferroni correction for multiple testing) are indicated with an asterisk (*); all other age and lgG comparisons had $p$-values < 0.001.

\section{RESULTS}

\section{Selection of Antibodies Associated With Cumulative Malaria Exposure}

In the HH-Artibonite survey, antibody levels, $\log _{10}(\mathrm{MFI})$ 's, to most antigens showed weak positive associations with increasing age (Spearman correlation coefficient, $r \leq 0.40$; Figure 1). AMA1 and MSP- $1_{19}$ showed moderate correlation with age (0.47 and 0.43, $p<0.001)$. The correlation coefficient between AMA-1 and MSP- $1_{19}$ was $0.67, p<0.001$ (Supplementary Figure 2). Together with GLURP-R2, these antigens were consistently the top three in each survey, although MSP-1 19 showed a weaker correlation $(0.35, p<0.001)$ than the other two antigens $(\geq 0.38$, $p<0.001)$ in the EAG-Grand'Anse survey. The validation of AMA-1 and MSP-1 19 as the most appropriate markers of cumulative exposure is consistent with the literature across multiple settings (2, 40-42). A strong age-related increase in seroprevalence to the combined metric of cumulative exposure (i.e., seropositivity to either AMA-1 or MSP-1 ${ }_{19}$ ) was seen across all surveys (chi-squared test for trend in proportions, $p<0.001$ ).

\section{Selection of Antibodies Associated With Current or Recent Malaria Exposure}

Antibody levels to the remaining 14 targets were included to predict hsRDT status using ROCs and a training dataset consisting of two-thirds of the EAG-Grand'Anse participants. Etramp 5 ag 1 had the highest AUC for predicting hsRDT status (0.892), followed by GLURP-R0 (0.825); Figure 2. Responses to the remaining antigens had an $\mathrm{AUC} \leq 0.8$. For all antigens except GLURP-R0, the AUC for children aged 1 to 15 was greater than that for individuals aged 16 or older. There were clear differences in AUCs between these age groups for some antibodies (e.g., HRP-2 and HSP40) while for others AUCs were similar (e.g., LSA-1 and rCSP). For children aged 15 or younger-in addition to Etramp 5 ag 1 and GLURP-R0SBP-1, Etramp 4 ag 2, HSP40 ag 1 and EBA-175 RIII-V had an $\mathrm{AUC}>0.8$.
The performance of antibodies to Etramp 5 ag 1 and GLURPR0 as accurate markers for hsRDT status (i.e., $\mathrm{AUC}>0.8$ ) was confirmed in the test dataset (Figure 3) as well as the $\mathrm{HH}$ - and EAG-Artibonite surveys (Supplementary Figure 3). Using seropositivity, for all age categories, Etramp 5 ag 1 showed similar performance compared to GLURP-R0 (all ages: $p=0.118$; children aged 1 to $15: p=0.060$; individuals aged 16 or older: $p=0.686)$ and the combined metric, representing seropositivity to either Etramp 5 ag 1 or GLURPR0 $(p=0.266 ; p=0.257 ; p=0.733)$. As no improvement was seen by adding GLURP-R0, Etramp 5 ag 1 alone was selected to represent recent exposure to falciparum malaria. Age-specific hsRDT and seroprevalence per survey is shown in Figure 4.

\section{DISCUSSION}

This study used multiplex serological data to select antigenspecific antibody responses associated with cumulative and current or recent malaria exposure in Haiti. IgG responses from almost 30,000 participants were analyzed from three surveys across two locations with differing levels of malaria transmission intensity. Results showed that IgG responses to antigens AMA-1 and MSP- $1_{19}$ best reflected cumulative exposure over time, whilst those to Etramp 5 ag 1 showed the highest accuracy in predicting current or recent infection when compared to hsRDT status. For the latter, survey data was used from the area experiencing the highest malaria transmission (Grand'Anse).

Continuous antibody responses to AMA-1 and MSP-1 19 were confirmed as the optimum representation of cumulative malaria exposure as they showed the strongest correlation with age. These antigens have been used extensively in serosurveillance for P. falciparum transmission (2). Seroconversion rates to AMA-1 and/or MSP- $1_{19}$ have shown a strong correlation with household altitude (42), EIR (2, 42), child mortality (43, 


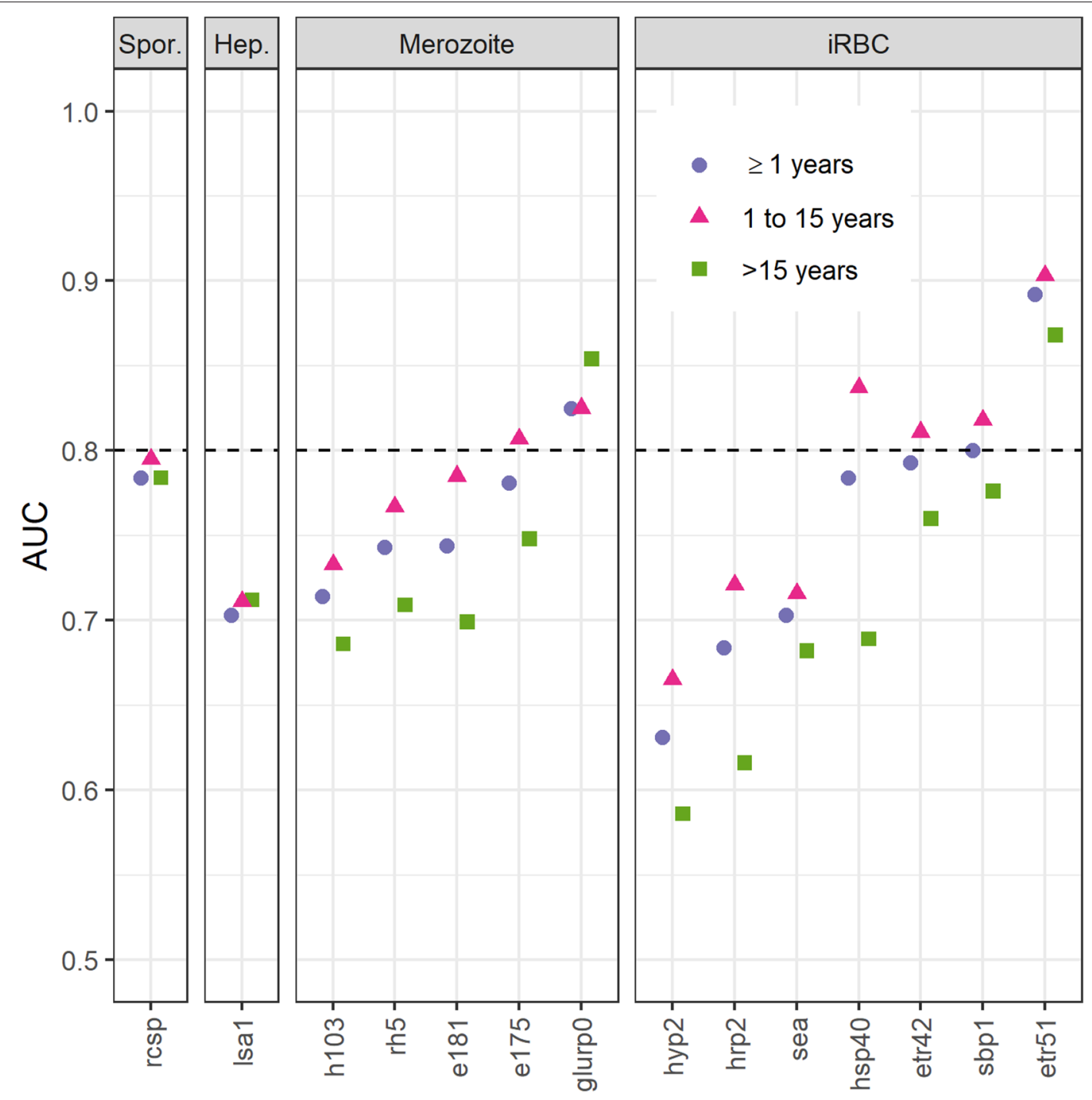

FIGURE 2 | Area under the curve (AUC) of receiver operating characteristic curves for immunoglobulin G (IgG) responses to fourteen Plasmodium falciparum antigens using highly sensitive rapid diagnostic test (hsRDT) status as the gold standard. Antigens (x-axis) are ordered by parasite life cycle stage and AUC value for all ages. IgG responses (IgG) in two-thirds of all EAG-Grand'Anse participants were used (i.e., the training dataset; $n=3,201)$ including 227 hsRDT positives (7.1\%). IgG responses were defined as log10-transformed median fluorescence intensities corrected for background reactivity. A threshold of 0.8 (dashed horizontal line) was used to select antigens for confirmation of results in the remaining one-third of the EAG-Grand'Anse dataset (see Figure 3). Spor.: sporozoite; Hep.: infected hepatocyte; iRBC: infected red blood cell.

44), the effect of malaria control interventions (43,45), and patterns in malaria transmission over time (44). In addition, GLURP-R2 was moderately correlated with age. GLURP-R2 has been associated with protection from (symptomatic) malaria $(46,47)$ as well as cumulative exposure $(40,48)$. This study shows strong indications that seropositivity to AMA-1, MSP$1_{19}$, and to a lesser extent, GLURP-R2, represent cumulative exposure in this Haitian population. Some previous studies have found that AMA-1 and MSP-1 19 can be good markers of active and recent infection in children $(7,8,41)$, though at higher transmission, even in children, repeated exposure and subsequent antibody boosting limits their use as recent exposure markers (9). Given that we have confirmed the appropriateness of AMA-1 and MSP-1 $_{19}$ as a cumulative marker of exposure in Haiti, there is an added benefit of enabling comparisons of results across malaria-endemic settings due to their extensive use in sero-surveillance to date $(2,40-42)$.

Next, continuous antibody responses were used to validate markers of current or recent $P$. falciparum exposure according to hsRDT results. Etramp 5 ag 1 and GLURP-R0 were found to be most accurate in predicting hsRDT status in 

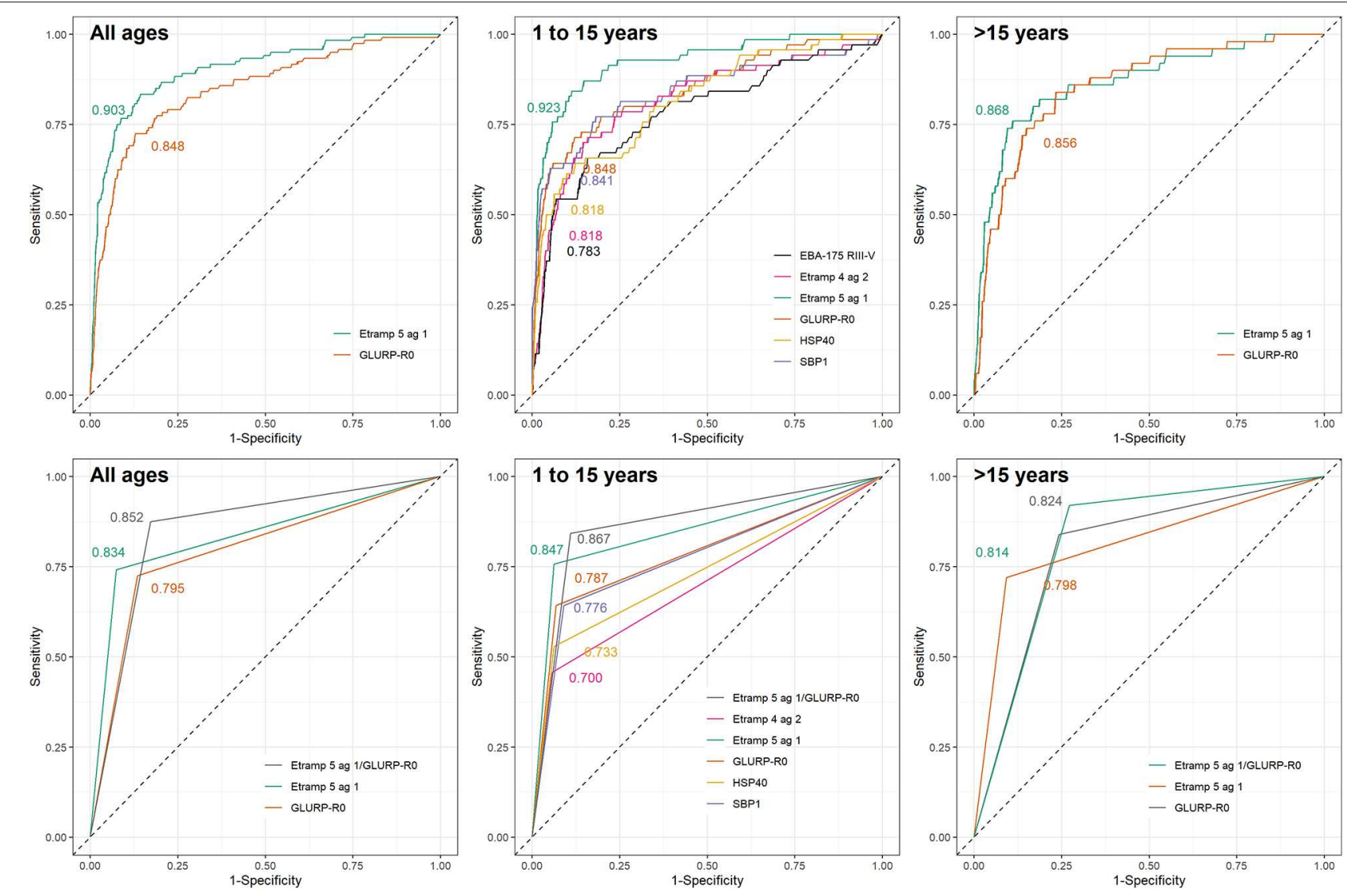

FIGURE 3 | Receiver operating characteristic curves for immunoglobulin G (IgG) responses to selected Plasmodium falciparum antigens using highly sensitive rapid diagnostic test (hsRDT) status as the gold standard. IgG responses in one-thirds of all EAG-Grand'Anse participants were used (i.e., the test dataset; $n=1,648$ ) including 120 hsRDT positives (7.3\%). IgG responses were defined as log10-transformed median fluorescence intensities corrected for background reactivity. Results are shown for continuous IgG responses (top) for antigens with an area-under-the-curve (AUC) $>0.8$ in the training dataset (Figure 2). Seropositivity results (bottom) are only shown for antigens with an AUC >0.8 using continuous IgG responses in the test dataset (top). AUC values are depicted on each plot. From left to right results are shown for: all ages, children aged 1 to 15 years and individuals older than 15 years.

the EAG-Grand'Anse survey. As no further improvement was seen by combining seropositivity to GLURP-R0 and Etramp 5 ag 1, seropositivity to Etramp 5 ag 1 alone was selected as representative of current or recent exposure to malaria. For children - in addition to Etramp 5 ag 1 and GLURP-R0 - SBP1, Etramp 4 ag 2, HSP40 ag 1, and EBA-175 RIII-V showed high accuracy in predicting hsRDT status. Intuitively, antibody responses in young children are more likely to represent a recent infection as they are unlikely to have developed a robust memory response following multiple infections (49), especially in areas of low transmission. Here we showed that all antibodies tested, except those to GLURP-R0, had higher accuracy in predicting hsRDT status in children compared to individuals aged 16 or older. Ideally, an IgG antibody response representing recent exposure does so across all ages as it minimizes the antigen panel needed. IgM antibody responses have regularly been suggested as a measure of recent exposure following their rapid acquisition and decline in viral infections, however, recent data showed IgM responses to malaria persisted over time and were not more short-lived than IgG responses to merozoite antigens (50).
Age-specific patterns of recent and cumulative exposure were consistent with expected exposure histories in the two transmission settings in Haiti: (1) recent exposure was higher in EAG-Grand'Anse compared to EAG-Artibonite which corresponds with the 2017 malaria incidence estimated at 18.1 and 0.6 per 1,000 inhabitants in Grand'Anse and Artibonite, respectively (source: National Malaria Control Program, PNCM, Haiti); (2) recent exposure was higher in healthcare-seeking populations compared to (likely asymptomatic) children attending school in Grand'Anse; (3) recent exposure was similar in children visiting a health facility or attending school in Artibonite, indicating that in this low transmission setting, healthcare-seeking is likely not driven by malaria disease; (4) cumulative and recent exposure was lowest in the $\mathrm{HH}$-Artibonite survey representing an asymptomatic, community-based population across all ages.

The gold standard for identification of antibodies with short (or longer) half-lives, is assessment of antibody acquisition and decay rates in longitudinal studies following naturally acquired malaria infections. However, such studies are costly, time and 


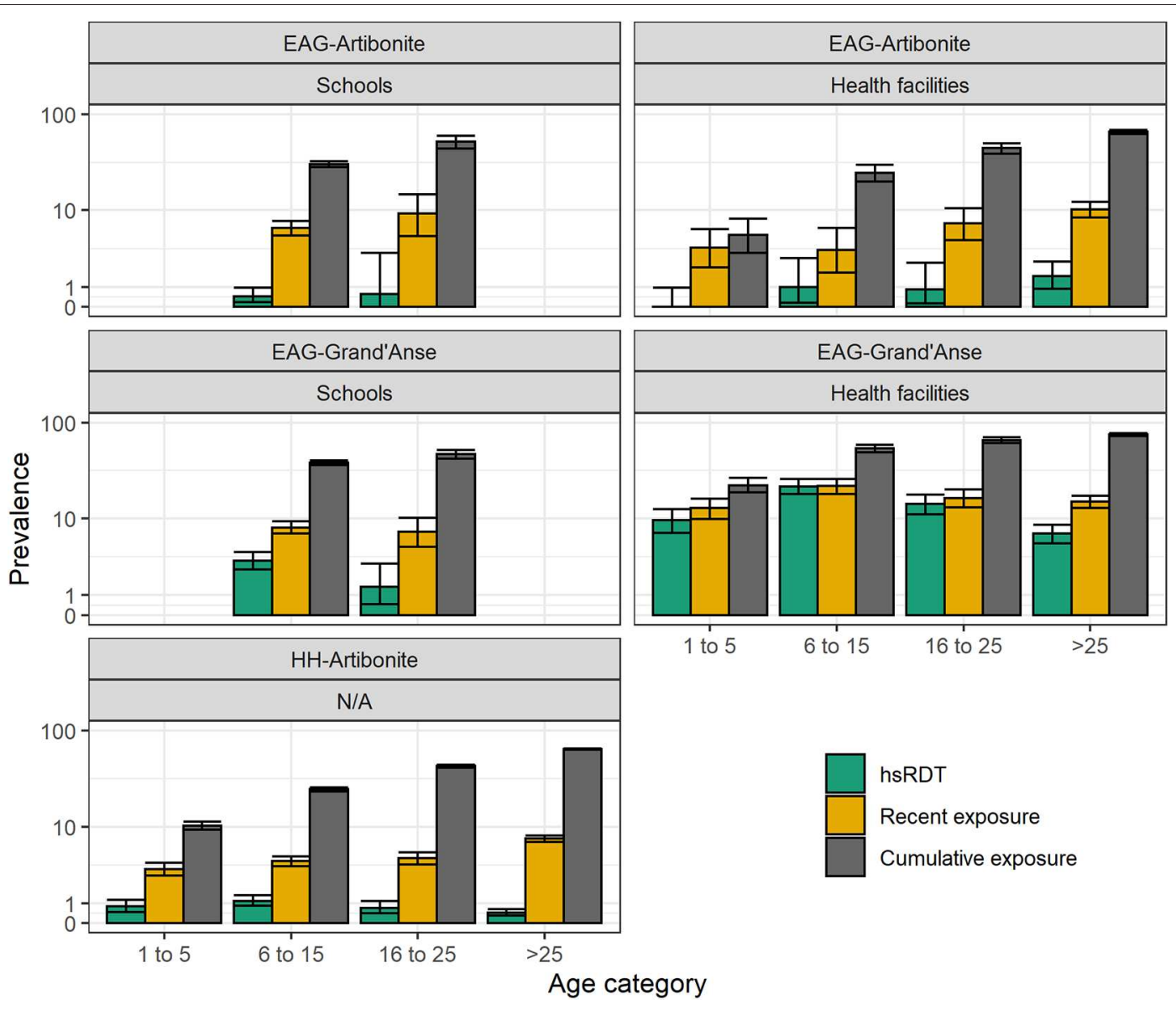

FIGURE 4 | Highly sensitive rapid diagnostic test (hsRDT) prevalence as well as recent and cumulative exposure seroprevalence per age category (in years) and survey in Haiti. Recent exposure represents seropositivity to early transcribed membrane protein 5 (Etramp 5 ag 1) while cumulative exposure represents seropositivity to apical membrane antigen 1 (AMA-1) and/or the $19 \mathrm{kDa}$ fragment of merozoite surface protein 1 (MSP-1 19$)$.

labor intensive, and thus rare. Moreover, no existing studies have used the same combination of the age range of the study population, antigen panel assessed and assay conditions (9, 25, 40). Here we used hsRDT data from cross-sectional surveys to inform the selection of current or recent exposure markers. It was assumed that the lack in specificity in using antibody levels to predict hsRDT status represented recent malaria infections that had become hsRDT negative. Alternatively, these could be malaria infections below the limit of detection of the hsRDT. The lack in sensitivity might represent infections that are too recently acquired for an antibody response to have developed. Alternatively, certain individuals may not produce detectable antibodies to a specific antigen following infection. The overlap in identified recent exposure markers with previous longitudinal studies [Etramp 5 in Helb et al. (9) and GLURP-R0 in Kerkhof et al. (25)] is promising for selection and standardization of antigen panels for sero-surveillance (51). However, Kerkhof et al. also identified GLURP-R2 and MSP-1 19 as recent exposure markers (25) - but these were better markers of cumulative exposure in our and other $(42-45,48)$ studies-indicating the need for caution and validation of these targets across populations and differing (histories of) transmission intensities. In settings with lower transmission, such as in Cambodia in the Kerkhof study (25), antibody levels are more likely to be reflective of recent exposure as they are not boosted following the lack of repeated exposure. This was also seen in our study, as nearly all antigen-specific antibodies analyzed accurately predicted hsRDT status in the Artibonite surveys where hsRDT prevalence was $<1 \%$.

There are different statistical techniques available to analyze multiplex antibody data. Boosted regression tree analysis has been used for $P$. vivax and $P$. knowlesi $(52,53)$. These studies showed that combined immune responses are more likely to be reflective of (recent) exposure though increments are small. It should be noted that the selection of antibodies for recent and cumulative exposure as described in this study is not meant to be exclusive. Remaining antibody responses may still be of interest for research-based studies interested in responses to, and patterns in, antibodies to specific targets. The antigens identified here were selected to enable rapid turnaround of analyses to directly inform malaria control programs in Haiti. 
Here we described the selection of antibodies associated with cumulative and recent malaria exposure in Haiti. We used antibody responses ( $\mathrm{IgG}$ ) to $23 \mathrm{P}$. falciparum recombinant proteins and peptides from 29,481 participants across three surveys. In the absence of a gold standard (i.e., longitudinal data), we used age and hsRDT status to make this selection. Seropositivity to AMA-1 and/or MSP- $1_{19}$ was selected to represent cumulative malaria exposure, while seropositivity to Etramp 5 ag 1 was selected to represent recent exposure to malaria. The identification of these antimalarial antibodies will simplify analyses and interpretation where results are needed to inform program decisions in Haiti.

\section{DATA AVAILABILITY STATEMENT}

The datasets generated for this study are available on request to the corresponding author.

\section{ETHICS STATEMENT}

The EAG-Artibonite and EAG-Grand'Anse surveys were approved by the London School of Hygiene and Tropical Medicine (LSHTM) Research Ethics Committee (10393), Tulane Institutional Review Board (794709) and the National Bioethics Committee in Haiti (1516-30). The Center for Global Health Associate Director of Science reviewed and approved the protocols; Centers for Disease Control and Prevention (CDC) investigators were not considered to be engaged in human subjects research (protocol 2016-135a). The HH-Artibonite survey was approved by the CDC Institutional Review Board (6821), LSHTM Research Ethics Committee (10466) and the National Bioethics Committee in Haiti (1516-29 and 1617-31).

For the EAG-Artibonite and EAG-Grand'Anse surveys, consent was collected from school directors following community meetings to inform parents and allow opt-out. At health facilities, adult participants provided consent directly, and consent for children ( $<18$ years) was provided by a parent or guardian and children above 6 years gave assent to participate. Individuals aged 16 or 17 who were married, head of household, or a parent were considered 'mature minors' and consented

\section{REFERENCES}

1. World Malaria Report. (2019). Available online at: https://www.who.int/ publications-detail/world-malaria-report-2019

2. Greenhouse B, Smith DL, Rodríguez-Barraquer I, Mueller I, Drakeley CJ. Taking sharper pictures of malaria with CAMERAs: combined antibodies to measure exposure recency assays. Am J Trop Med Hyg. (2018) 99:1120-7. doi: 10.4269/ajtmh.18-0303

3. Bosomprah S. A mathematical model of seropositivity to malaria antigen, allowing seropositivity to be prolonged by exposure. Malar J. (2014) 13:12. doi: 10.1186/1475-2875-13-12

4. Corran P, Coleman P, Riley E, Drakeley C. Serology: a robust indicator of malaria transmission intensity? Trends Parasitol. (2007) 23:575-82. doi: 10.1016/j.pt.2007.08.023

5. Yman V, White MT, Asghar M, Sundling C, Sondén K, Draper SJ, et al. Antibody responses to merozoite antigens after natural Plasmodium directly. Thumbprint consent or assent (countersigned by a witness) was used for illiterate participants. For the $\mathrm{HH}$ Artibonite survey, verbal consent for overall permission to conduct the survey was obtained from the head of household and/or primary caretaker and documented in the electronic data collection instrument. Individual-level verbal consent was sought from each present individual for the blood sample collection, and assent from persons aged 7-17 years.

\section{AUTHOR CONTRIBUTIONS}

JL, MC, TPE, TD, RA, GS, CD, KH, and ER designed the surveys. TPE, TD, RA, VJ, GS, KH, and MC performed and supported field data collection. KT, JGB, SS and ER provided antigen constructs. JP, IR, TE, and GM performed laboratory data collection. $\mathrm{LH}, \mathrm{AE}, \mathrm{JB}, \mathrm{KT}, \mathrm{CD}$, and ER supported laboratory data collection. LH, GS, KT, ER, and CD analyzed and interpreted the data. $\mathrm{LH}$ wrote the first draft of the manuscript with support from GS and CD. All authors read and approved the final manuscript.

\section{FUNDING}

This study was conducted by the Malaria Zero Alliance (http://malariazeroalliance.org/), funded by the Bill \& Melinda Gates Foundation through grant \#OPP1114297 to the CDC Foundation.

\section{ACKNOWLEDGMENTS}

The authors would like to thank all Malaria Zero field teams for performing data collection as well as the survey participants. We would like to acknowledge Professor Simon Draper and Dr. Jing Jin (Jenner Institute, University of Oxford, Oxford, United Kingdom) for provision of RH5.1 protein.

\section{SUPPLEMENTARY MATERIAL}

The Supplementary Material for this article can be found online at: https://www.frontiersin.org/articles/10.3389/fimmu. 2020.00928/full\#supplementary-material falciparum infection: kinetics and longevity in absence of re-exposure. $B M C$ Medicine. (2019) 17:22. doi: 10.1186/s12916-019-1255-3

6. Wipasa J, Suphavilai C, Okell LC, Cook J, Corran PH, Thaikla K, et al. Long-lived antibody and B Cell memory responses to the human malaria parasites, Plasmodium falciparum and Plasmodium vivax. PLoS Pathog. (2010) 6:e1000770. doi: 10.1371/journal.ppat.1000770

7. Mugyenyi CK, Elliott SR, Yap XZ, Feng G, Boeuf P, Fegan G, et al. Declining malaria transmission differentially impacts the maintenance of humoral immunity to Plasmodium falciparum in children. J Infect Dis. (2017) 216:88798. doi: 10.1093/infdis/jix370

8. Kinyanjui SM, Conway DJ, Lanar DE, Marsh K. IgG antibody responses to Plasmodium falciparum merozoite antigens in Kenyan children have a short half-life. Malaria J. (2007) 6:82. doi: 10.1186/1475-28 75-6-82

9. Helb DA, Tetteh KKA, Felgner PL, Skinner J, Hubbard A, Arinaitwe E, et al. Novel serologic biomarkers provide accurate estimates of recent Plasmodium 
falciparum exposure for individuals and communities. Proc Natl Acad Sci USA. (2015) 112:E4438-47. doi: 10.1073/pnas. 1501705112

10. Wu L, Hall $\mathrm{T}$, Ssewanyana I, Oulton $\mathrm{T}$, Patterson C, Vasileva $\mathrm{H}$, et al. Optimisation and standardisation of a multiplex immunoassay of diverse Plasmodium falciparum antigens to assess changes in malaria transmission using sero-epidemiology. Wellcome Open Res. (2019) 4:26. doi: 10.12688/wellcomeopenres.14950.1

11. Malaria Zero. Malaria Zero Alliance. Available online at: https://www. malariazeroalliance.org/malaria-zero

12. WHO. Country Profiles. WHO. Available online at: http://www.who.int/ malaria/publications/country-profiles/2017/en/

13. Druetz T, Andrinopoulos K, Boulos L-M, Boulos M, Noland GS, Desir L, et al. "Wherever doctors cannot reach, the sunshine can": overcoming potential barriers to malaria elimination interventions in Haiti. Malar J. (2018) 17:393. doi: 10.1186/s12936-018-2553-5

14. Rogier E, Hamre KES, Joseph V, Plucinski MM, Presume J, Romilus I, et al. Conventional and high-sensitivity malaria rapid diagnostic test performance in two transmission settings: Haiti 2017. J Infect Dis. (2019) 221:786-95. doi: 10.1093/infdis/jiz525

15. Hoogen LL van den, Présumé J, Romilus I, Mondélus G, Elismé T, Sepúlveda $\mathrm{N}$, et al. Quality control of multiplex antibody detection in samples from large-scale surveys: the example of malaria in Haiti. Sci Rep. (2020) 10:1-10. doi: 10.1038/s41598-020-57876-0

16. Druetz T, Stresman G, Ashton RA, van den Hoogen LL, Joseph V, Fayette C, et al. Programmatic Options for Monitoring Malaria in Elimination Settings: Easy Access Group Surveys to Investigate Plasmodium Falciparum Epidemiology in Two Regions With Differing Endemicity in Haiti. BMC Medicine (2020). in press. doi: 10.1186/s12916-020-01611-z

17. Kastenmüller K, Espinosa DA, Trager L, Stoyanov C, Salazar AM, Pokalwar $\mathrm{S}$, et al. Full-length Plasmodium falciparum circumsporozoite protein administered with long-chain poly $(\mathrm{I} \bullet \mathrm{C})$ or the Toll-like receptor 4 agonist glucopyranosyl lipid adjuvant-stable emulsion elicits potent antibody and CD4+ T cell immunity and protection in mice. Infect Immun. (2013) 81:789800. doi: 10.1128/IAI.01108-12

18. Plucinski MM, Candrinho B, Chambe G, Muchanga J, Muguande O, Matsinhe G, et al. Multiplex serology for impact evaluation of bed net distribution on burden of lymphatic filariasis and four species of human malaria in northern Mozambique. PLoS Negl Trop Dis. (2018) 12:e0006278. doi: 10.1371/journal.pntd.0006278

19. Raj DK, Nixon CP, Nixon CE, Dvorin JD, DiPetrillo CG, Pond-Tor S, et al. Antibodies to PfSEA-1 block parasite egress from RBCs and protect against malaria infection. Science. (2014) 344:871-7. doi: 10.1126/science.1254417

20. Grüring C, Heiber A, Kruse F, Ungefehr J, Gilberger T-W, Spielmann T. Development and host cell modifications of Plasmodium falciparum blood stages in four dimensions. Nat Commun. (2011) 2:165. doi: 10.1038/ncomms1169

21. Rogier E, Plucinski M, Lucchi N, Mace K, Chang M, Lemoine JF, et al. Bead-based immunoassay allows sub-picogram detection of histidinerich protein 2 from Plasmodium falciparum and estimates reliability of malaria rapid diagnostic tests. PLoS ONE. (2017) 12:e0172139. doi: 10.1371/journal.pone.0172139

22. Spielmann T, Fergusen DJP, Beck H-P. etramps, a new Plasmodium falciparum gene family coding for developmentally regulated and highly charged membrane proteins located at the parasite-host cell interface. Mol Biol Cell. (2003) 14:1529-44. doi: 10.1091/mbc.e02-04-0240

23. Polley SD, Conway DJ, Cavanagh DR, McBride JS, Lowe BS, Williams TN, et al. High levels of serum antibodies to merozoite surface protein 2 of Plasmodium falciparum are associated with reduced risk of clinical malaria in coastal Kenya. Vaccine. (2006) 24:4233-46. doi: 10.1016/j.vaccine.2005. 06.030

24. Taylor RR, Smith DB, Robinson VJ, McBride JS, Riley EM. Human antibody response to Plasmodium falciparum merozoite surface protein 2 is serogroup specific and predominantly of the immunoglobulin G3 subclass. Infect Immun. (1995) 63:4382-8. doi: 10.1128/IAI.63.11.4382-4388.1995

25. Kerkhof K, Sluydts V, Willen L, Kim S, Canier L, Heng S, et al. Serological markers to measure recent changes in malaria at population level in Cambodia. Malar J. (2016) 15:529. doi: 10.1186/s12936-016$1576-\mathrm{z}$
26. Theisen M, Vuust J, Gottschau A, Jepsen S, Høgh B. Antigenicity and immunogenicity of recombinant glutamate-rich protein of Plasmodium falciparum expressed in Escherichia coli. Clin Diagn Lab Immunol. (1995) 2:30-4. doi: 10.1128/CDLI.2.1.30-34.1995

27. Burghaus PA, Holder AA. Expression of the 19-kilodalton carboxy-terminal fragment of the Plasmodium falciparum merozoite surface protein-1 in Escherichia coli as a correctly folded protein. Mol Biochem Parasitol. (1994) 64:165-9. doi: 10.1016/0166-6851(94)90144-9

28. Pearce JA, Mills K, Triglia T, Cowman AF, Anders RF. Characterisation of two novel proteins from the asexual stage of Plasmodium falciparum, H101 and H103. Mol Biochem Parasitol. (2005) 139:141-51. doi: 10.1016/j.molbiopara.2004.09.012

29. Richards JS, Stanisic DI, Fowkes FJI, Tavul L, Dabod E, Thompson JK, et al. Association between naturally acquired antibodies to erythrocyte-binding antigens of Plasmodium falciparum and protection from malaria and highdensity parasitemia. Clin Infect Dis. (2010) 51:e50-60. doi: 10.1086/656413

30. Collins CR, Withers-Martinez C, Bentley GA, Batchelor AH, Thomas AW, Blackman MJ. Fine mapping of an epitope recognized by an invasion-inhibitory monoclonal antibody on the malaria vaccine candidate apical membrane antigen 1. J Biol Chem. (2007) 282:7431-41. doi: 10.1074/jbc.M6105 62200

31. Reiling L, Richards JS, Fowkes FJI, Barry AE, Triglia T, Chokejindachai $\mathrm{W}$, et al. Evidence that the erythrocyte invasion ligand PfRh2 is a target of protective immunity against Plasmodium falciparum malaria. J Immunol. (2010) 185:6157-67. doi: 10.4049/jimmunol.10 01555

32. Stubbs J, Simpson KM, Triglia T, Plouffe D, Tonkin CJ, Duraisingh MT, et al. Molecular mechanism for switching of $P$. falciparum invasion pathways into human erythrocytes. Science. (2005) 309:1384-7. doi: 10.1126/science.111 5257

33. Jin J, Tarrant RD, Bolam EJ, Angell-Manning P, Soegaard M, Pattinson DJ, et al. Production, quality control, stability, and potency of cGMP-produced Plasmodium falciparum RH5.1 protein vaccine expressed in Drosophila S2 cells. NPJ Vaccines. (2018) 3:32. doi: 10.1038/s41541-018-0071-7

34. Rogier E, van den Hoogen L, Herman C, Gurrala K, Joseph V, Stresman G, et al. High-throughput malaria serosurveillance using a one-step multiplex bead assay. Malar J. (2019) 18:402. doi: 10.1186/s12936-019-3027-0

35. R Core Team. R: A Language and Environment for Statistical Computing. Vienna: R Foundation for Statistical Computing (2017). Available online at: https://www.R-project.org/

36. Wei T, Simko V. R package "corrplot": Visualization of a Correlation Matrix (Version 0.84). (2017). Available online at: https://github.com/taiyun/corrplot

37. Plucinski MM, McElroy PD, Dimbu PR, Fortes F, Nace D, Halsey ES, et al. Clearance dynamics of lactate dehydrogenase and aldolase following antimalarial treatment for Plasmodium falciparum infection. Parasit Vectors. (2019) 12:293. doi: 10.1186/s13071-019-3549-x

38. Malaria Policy Advisory Committee Meeting. WHO Technical Consultation on Research Requirements to Support Policy Recommendations on Highly Sensitive Point-Of-Care Diagnostics for P. falciparum Malaria. Geneva: World Health Organization (2018)

39. Robin X, Turck N, Hainard A, Tiberti N, Lisacek F, Sanchez J-C, et al. pROC: an open-source package for R and S+ to analyze and compare ROC curves. BMC Bioinform. (2011) 12:77. doi: 10.1186/1471-2105-12-77

40. Ondigo BN, Hodges JS, Ireland KF, Magak NG, Lanar DE, Dutta S, et al. Estimation of recent and long-term malaria transmission in a population by antibody testing to multiple Plasmodium falciparum antigens. J Infect Dis. (2014) 210:1123-32. doi: 10.1093/infdis/jiu225

41. McCallum FJ, Persson KEM, Fowkes FJI, Reiling L, Mugyenyi CK, Richards JS, et al. Differing rates of antibody acquisition to merozoite antigens in malaria: implications for immunity and surveillance. J Leukoc Biol. (2017) 101:913-25. doi: 10.1189/jlb.5MA0716-294R

42. Drakeley CJ, Corran PH, Coleman PG, Tongren JE, McDonald SLR, Carneiro I, et al. Estimating medium- and long-term trends in malaria transmission by using serological markers of malaria exposure. Proc Natl Acad Sci USA. (2005) 102:5108-13. doi: 10.1073/pnas.0408725102

43. Cook J, Kleinschmidt I, Schwabe C, Nseng G, Bousema T, Corran PH, et al Serological markers suggest heterogeneity of effectiveness of malaria control 
interventions on Bioko Island, equatorial Guinea. PLoS ONE. (2011) 6:e25137. doi: 10.1371/journal.pone.0025137

44. van den Hoogen LL, Griffin JT, Cook J, Sepúlveda N, Corran P, Conway DJ, et al. Serology describes a profile of declining malaria transmission in Farafenni, The Gambia. Malar J. (2015) 14:416. doi: 10.1186/s12936-015-0939-1

45. Cook J, Reid H, Iavro J, Kuwahata M, Taleo G, Clements A, et al. Using serological measures to monitor changes in malaria transmission in Vanuatu. Malar J. (2010) 9:169. doi: 10.1186/1475-2875-9-169

46. Kana IH, Singh SK, Garcia-Senosiain A, Dodoo D, Singh S, Adu B, et al. Breadth of functional antibodies is associated with Plasmodium falciparum merozoite phagocytosis and protection against febrile malaria. J Infect Dis. (2019) 220:275-84. doi: 10.1093/infdis/ jiz088

47. Adu B, Cherif MK, Bosomprah S, Diarra A, Arthur FKN, Dickson EK, et al. Antibody levels against GLURP R2, MSP1 block 2 hybrid and AS202.11 and the risk of malaria in children living in hyperendemic (Burkina Faso) and hypo-endemic (Ghana) areas. Malar J. (2016) 15:123. doi: 10.1186/s12936-016-1146-4

48. Björkman A, Shakely D, Ali AS, Morris U, Mkali H, Abbas AK, et al. From high to low malaria transmission in Zanzibar-challenges and opportunities to achieve elimination. BMC Medicine. (2019) 17:14. doi: 10.1186/s12916-018-1243-z

49. White MT, Griffin JT, Akpogheneta O, Conway DJ, Koram KA, Riley EM, et al. Dynamics of the antibody response to Plasmodium falciparum infection in African children. J Infect Dis. (2014) 210:1115-22. doi: 10.1093/infdis/jiu219

50. Boyle MJ, Chan JA, Handayuni I, Reiling L, Feng G, Hilton A, et al. IgM in human immunity to Plasmodium falciparum malaria. Sci Adv. (2019) 5:eaax4489. doi: 10.1126/sciadv.aax4489
51. Arnold BF, Scobie HM, Priest JW, Lammie PJ. Integrated serologic surveillance of population immunity and disease transmission. Emerging Infect Dis. (2018) 24:1188-94. doi: 10.3201/eid2407.171928

52. Fornace KM, Brock PM, Abidin TR, Grignard L, Herman LS, Chua TH, et al. Environmental risk factors and exposure to the zoonotic malaria parasite Plasmodium knowlesi across northern Sabah, Malaysia: a populationbased cross-sectional survey. Lancet Planet Health. (2019) 3:e179-86. doi: 10.1016/S2542-5196(19)30045-2

53. Longley RJ, White MT, Takashima E, Brewster J, Morita M, Harbers M, et al. Development and validation of serological markers for detecting recent exposure to Plasmodium vivax infection. bioRxiv 481168. (2018). doi: $10.1101 / 481168$

Disclaimer: The findings and conclusions presented in this report are those of the authors and do not necessarily reflect the official position of the Centers for Disease Control and Prevention.

Conflict of Interest: The authors declare that the research was conducted in the absence of any commercial or financial relationships that could be construed as a potential conflict of interest.

Copyright (C) 2020 van den Hoogen, Stresman, Présumé, Romilus, Mondélus, Elismé, Existe, Hamre, Ashton, Druetz, Joseph, Beeson, Singh, Boncy, Eisele, Chang, Lemoine, Tetteh, Rogier and Drakeley. This is an open-access article distributed under the terms of the Creative Commons Attribution License (CC BY). The use, distribution or reproduction in other forums is permitted, provided the original author(s) and the copyright owner(s) are credited and that the original publication in this journal is cited, in accordance with accepted academic practice. No use, distribution or reproduction is permitted which does not comply with these terms. 